\title{
The absence of jets in cataclysmic variable stars ${ }^{\star}$
}

\author{
N. Soker ${ }^{1}$ and J.-P. Lasota ${ }^{2}$ \\ 1 Department of Physics, Technion-Israel Institute of Technology, 32000 Haifa, Israel \\ e-mail: soker@physics.technion.ac.il \\ 2 Institut d'Astrophysique de Paris, 98bis boulevard Arago, 75014 Paris, France \\ e-mail: lasota@iap.fr
}

Received 7 March 2004 / Accepted 20 April 2004

\begin{abstract}
We show that the recently developed thermal model which successfully describes how jets are launched by young stellar objects, when applied to system containing disk-accreting white dwarfs naturally explain the otherwise surprising absence of jets in cataclysmic variable stars. Our main argument uses the crucial element of the thermal model, namely that the accreted material is strongly shocked due to large gradients of physical quantities in the boundary layer, and then cools on a time scale longer than its ejection time from the disk. In our scenario the magnetic fields are weak, and serve only to recollimate the outflow at large distances from the source, or to initiate the shock, but not as a jet-driving agent. Using two criteria in that model, for the shock formation and for the ejection of mass, we find the mass accretion rate above which jets could be blown from accretion disks around young stellar objects and white dwarfs. We find that these accretion mass rates are $\dot{M}($ YSO $) \gtrsim 10^{-7} M_{\odot} \mathrm{yr}^{-1}$ and $\dot{M}(\mathrm{WD}) \gtrsim 10^{-6} M_{\odot} \mathrm{yr}^{-1}$ for young stellar objects and white dwarfs respectively. Considering the uncertainties of the model, these limits could overestimate the critical value by a factor of $\sim 10$.
\end{abstract}

Key words. accretion, accretion disks - ISM: jets and outflows - stars: winds, outflows - stars: novae, cataclysmic variables

\section{Introduction}

It is widely believed that most astrophysical jets, and all massive jets (to distinguish from low density hot-plasma jets from radio pulsars) are launched from accretion disks (Livio 1999, 2000a). This belief is supported by observations of jets in Young Stellar Object (YSOs), Low-Mass and HighMass X-ray Binaries (LMXBs \& HMXBs) and Active Galactic Nuclei (AGNs) which all are systems containing accretion disks (or at least accretion flows with a considerable amount of angular momentum). The apparent universality of the accretion disk-jet relation is spoiled by one class of systems: Cataclysmic Variables (CVs). They are close binary systems in which a white dwarf accretes matter lost by its Roche-lobe filling low-mass companion (see Warner 1995 for a review). For weak enough white-dwarf's magnetic fields CVs posses accretion disks. But no jets have ever been observed from these numerous and extremely well observed binaries. The reported occurrence (Shahbaz et al. 1997) failed to be confirmed (O'Brien $\&$ Cohen 1998) and the system itself is most probably not a CV but a Super Soft X-ray Source (SSXS; Knigge et al. 2000). Jets have been reported after the outburst of the fast nova V1494 Aql (Iijima \& Esenoglu 2003). As we will mention later, the mass accretion rate during this stage was very high (Retter, A., private communication 2004). However, jets

^ Research supported by the Israel Science Foundation, and the Centre National d'Études Spatiales. have been observed emanating from some other SSXSs (see below) which shows that the absence of jets in CVs cannot be attributed to some special properties of white dwarfs in binary systems since white dwarfs are also present in SSXSs, and symbiotic systems, some of which blow jets. We do not find very compelling the suggestion by Knigge \& Livio (1998) that jets in CVs have very weak optical features, and could therefore have eluded detection, even if they exist. We rather assume that the absence of jets in CVs is real.

One can expect that the absence of jets in CVs could tell us something about the still mysterious jets launching mechanism. Commonly it is assumed that magnetic fields play crucial roles in the formation of jets. Magnetic fields can appear in three types of roles:

(1) In triggering the jet ejection events, e.g., by causing instabilities in the disk. These types of "magnetospheric" MHD instabilities could exist in accretion disks even when the central star has no magnetic field (e.g., Li \& Narayan 2004, and references therein). MHD instabilities, turbulence, or other disturbances may lead to strong shocks; the high post-shock pressure may accelerate gas and form jets and/or winds, e.g., as was shown for non-radiative accretion around a black hole by De Villiers et al. (2004).

(2) In accelerating the jets. There are many models and countless of papers on this subject. Basically, most models are based on the operation of large scale magnetic fields 
driving the flow from the disk; either via the "centrifugal wind" mechanism, first proposed by Blandford \& Payne (1982), or from a narrow region in the magnetopause of the stellar field via an "X-wind mechanism" introduced by Shu et al. (1988, 1991) and in a somewhat different setting, by Ferreira \& Pelletier (1993, 1995). See recent reviews by Königl \& Pudritz (2000), Shu et al. (2000) and Ferreira (2002). It should, however, be pointed out that the origin of the large scale magnetic fields and the manner that open field lines of sufficiently strong magnitude persist (in the centrifugal wind models), or the manner by which a stellar field interacts with the disk, allowing inflow and at the same time driving an outflow (in the $\mathrm{X}$-wind models) are still open key issues of the theory (e.g., Heyvaerts et al. 1996). In addition, it seems that thermal pressure is needed in some of these models (e.g., Ferreira \& Casse 2004).

(3) In collimating the jets (e.g., Heyvaerts \& Norman 1989). The collimation issue is, however, still quite controversial and while magnetic collimation is certainly plausible, its exact nature is probably quite involved and still not fully understood (see the recent works of Bogovalov \& Tsinganos 2001 and Li 2002).

Magnetic jet launching models fail to account for the absence of jets in CVs despite of some interesting suggestions in Livio (2000b). It is therefore justified to consider jet launching mechanisms in which magnetic fields would be deprived of at least one of the three roles. In the present article we show that the absence of jets in CVs is naturally explained by the model of thermal pressure acceleration proposed by Torbett (1984) and Torbett \& Gilden (1992), and which was developed and extended recently by Soker \& Regev (2003; hereafter SR03) to explain collimated outflows in YSO.

SR03 interest in thermal pressure acceleration of jets was motivated by new results from recent X-ray observations of YSO. These show that there is essentially no difference between the properties of X-ray emission from YSO with and without outflows (Getman et al. 2002), imposing quite severe constraints on models based on magnetic launching of jets. In the thermally-driven jet model, the magnetic fields are weak, and serve only to re-collimate the outflow at large distances from the source, (role (3) above), and possibly trigger disturbances in the boundary layer (BL), (role (1) above). The BL is the inner layer, where the disk adjust itself to the conditions of the accreting star. A crucial ingredient of the model is that the accreted material is strongly shocked, and that it cools down on a time scale longer than its ejection time from the inner disk. SR03 find that the thermal acceleration mechanism works only when the accretion rate in YSO accretion disk is large enough and the $\alpha$ parameter of the disk small enough - otherwise the cooling time is too short and significant ejection does not take place. SR03 term these strong shocks "spatiotemporally localized (but not too small!) accretion shocks", or SPLASHes.

In the present paper we extend the analysis of SR03 to white dwarfs (WDs) accretors. We compare the derived conditions for thermally launching jets from accretions disks around YSO and around WDs. YSO refers also to main sequence stars accreting from mass-losing companions stars. We show that the thermally-driven jet model can be extended to jets blown by disks around WDs and explain the absence of jets in CVs.

\section{The opacity mechanism}

There are two basic radiative cooling time-scales, which lead to two conditions for launching of strong outflows by thermal pressure. The first is that the photon-diffusion cooling time of the entire BL region, $\tau_{\text {diff }}$, be longer than the ejection time of the jet $\tau_{\mathrm{ej}}$. This applies to the jet-acceleration phase, after the disk-material has passed through a strong shock. In other words, this is the condition for the SPLASH to be able to eject shocked material. The second time scale refers to the buildup phase of a strong shock, i.e. determines the condition for the SPLASH formation. In the following sections we will consider the constrains on these two cooling time-scales,

The photon diffusion time-scales depend on the opacity, which itself strongly depends on the density and temperature (e.g., Rogers \& Iglesias 1992; Seaton et al. 1994). In Fig. 1 we plot the mean Rosseland opacity coefficient as a function of the temperature for four values of the density. The opacity is taken from Seaton et al. (1994), for a composition of $X=0.7$ and $Z=0.03$. We take a little higher than solar metallicity to account for enrichment in presently formed YSOs.

The temperature range characterizes accretion disks and BLs around YSOs is $\sim 10^{4}-10^{6} \mathrm{~K}$, while around a WD accretor this range is $\sim 10^{6}-10^{8} \mathrm{~K}$. From Fig. 1 it appears immediately that the opacity behaves markedly different in these two ranges. We will explore the significance of this difference in the following sections. The opacity coefficient in the relevant range for YSO accretors can be adequately fitted by

$$
\begin{aligned}
\log \kappa= & 1+0.7 \log \left(\frac{\rho}{10^{-7}}\right)-2 \log \left(\frac{T}{10^{5}}\right) \\
& -0.6 \log \left(\frac{\rho}{10^{-7}}\right) \log \left(\frac{T}{10^{5}}\right),
\end{aligned}
$$

where $\kappa$ is the opacity coefficient and $\rho$ the density in the disk; all quantities being expressed in cgs units. For later calculations it will be convenient to write the opacity as

$\kappa=10\left(\frac{\rho}{10^{-7}}\right)^{0.7}\left(\frac{T}{10^{5}}\right)^{-2}\left(\frac{\rho}{10^{-7}}\right)^{-0.6 \log \left(T / 10^{5}\right)}$.

The last two approximations hold for $-8 \lesssim \log \rho \lesssim-5$ and $4.5 \lesssim \log T \lesssim 5.5$.

\section{The acceleration phase}

The radiative diffusion time from the entire $\mathrm{BL}$ region depends on the conditions in the disk BL and can be determined from the photon mean free path $\lambda$, and the size of the region from which the photons have to escape. It is assumed that the gas has been strongly shocked. Using formulae from Torbett (1984), Torbett \& Gilden (1992) and SR03, one can write the diffusion time as

$\tau_{\mathrm{diff}}=\epsilon^{2} R^{2} \frac{\rho \kappa}{c}$ 


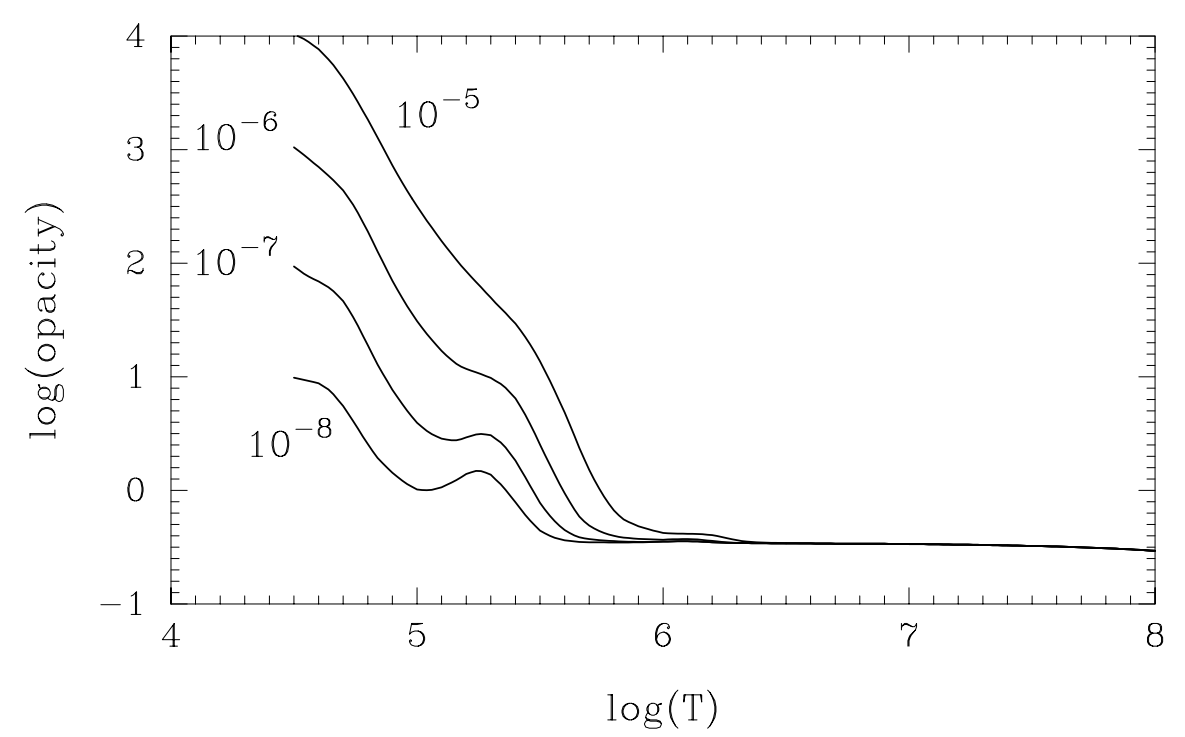

Fig. 1. Opacity as a function of temperature for four values of the density as indicated, and for a composition of $X=0.7$ and $Z=0.03$. Quantities are given in cgs units.

where $\epsilon=H / R, H$ is the vertical disk's scale height, $R$ is the distance from the center of the disk, and $c$ is the speed of light. We will use the expression for the density in an $\alpha$-disk model in which the radial velocity of the slowly accreting mass is given by (Pringle 1981) $v_{\mathrm{r}} \simeq \alpha \epsilon^{2} v_{\mathrm{K}}$, where $\alpha$ is the disk-viscosity parameter, and $v_{\mathrm{K}}$ the Keplerian tangential velocity. From the mass conservation equation $\dot{M}=2 \pi R 2 H \rho v_{\mathrm{r}}$, one finds

$\rho=\frac{\dot{M}}{4 \pi \alpha \epsilon^{3} R^{2} v_{\mathrm{K}}}$.

Taking into account that behind a strong shock $\rho$ increases by a factor four, one gets for the diffusion time

$\tau_{\text {diff }}=\frac{\dot{M} \kappa}{\pi c \alpha \epsilon v_{\mathrm{K}}}$.

This has to be compared with the dynamical ejection time $\tau_{\mathrm{ej}}=$ $H / v_{\text {esc }}=\epsilon R / v_{\text {esc }}$. Taking $v_{\mathrm{K}} \simeq 0.7 v_{\text {esc }}$ one has

$$
\begin{aligned}
\frac{\tau_{\text {diff }}}{\tau_{\text {ej }}} \simeq \frac{\dot{M} \kappa}{\pi c \alpha \epsilon^{2} R} \simeq & 1.3\left(\frac{\epsilon}{0.1}\right)^{-2}\left(\frac{\alpha}{0.1}\right)^{-1}\left(\frac{\dot{M}}{10^{-7} M_{\odot} \mathrm{yr}^{-1}}\right) \\
& \times\left(\frac{\kappa}{\mathrm{cm}^{2} \mathrm{~g}^{-1}}\right)\left(\frac{R}{R_{\odot}}\right)^{-1} .
\end{aligned}
$$

For a SPLASH to be able to eject a collimated outflow the ejection time $\tau_{\mathrm{ej}}$ must be shorter than the radiation diffusion time $\tau_{\text {diff }}$ (SR03). For the strong shocks the temperature is $T \gtrsim 10^{6}$ (see below), and $\kappa \simeq 0.4$, for both YSOs and WDs. The condition $\tau_{\text {diff }} \gtrsim \tau_{\text {ej }}$ is met for YSOs, $R \simeq 1 R_{\odot}$, accreting at a rate of

$\dot{M}_{\mathrm{S}} \gtrsim 2 \times 10^{-7}\left(\frac{\epsilon}{0.1}\right)^{2}\left(\frac{\alpha}{0.1}\right) M_{\odot} \mathrm{yr}^{-1} \quad$ for $\quad$ YSOs,

whereas for WDs $\left(R \simeq 0.01 R_{\odot}\right)$ it requires

$\dot{M}_{\mathrm{s}} \gtrsim 2 \times 10^{-9}\left(\frac{\epsilon}{0.1}\right)^{2}\left(\frac{\alpha}{0.1}\right) M_{\odot} \mathrm{yr}^{-1} \quad$ for WDs,

which means that for both types of objects if a SPLASH forms it would be able to eject collimated flows. We turn now to the second cooling time-scale which determines when SPLASH can form.

\section{The buildup of a strong shock}

The SPLASH model assumes that hundreds of small blobs are formed in the sheared BL (Sect. 2 of SR03). The blobs occasionally collide with each other, and create shocks which cause the shocked regions to expand in all directions. If the shocked regions continue to expand out into the path of yet more circulating blobs, stronger shocks may be created, as was proposed by Pringle \& Savonije (1979) to explain the emission of X-rays out of disk BLs in dwarf novae. For the shocked blobs to expand, the radiative cooling time of individual blobs, $t_{\text {cool }} \simeq \ell^{2} \kappa \rho_{\mathrm{b}} / c$, must be longer than the adiabatic expansion time of individual blobs $t_{\mathrm{ad}}=\ell / c_{\mathrm{s}}$, where $\ell$ is the size of an expanding blob, and $\rho_{\mathrm{b}}$ the post-shock blob's density. SR03 demand also that the blobs be small, because the dissipation time of disk material to form the SPLASH must be shorter than the jet ejection time (Eq. (24) of SR03). This gives for the blobs size $\ell \sim \epsilon^{2} R$ (Eq. (27) of SR03). The condition of long cooling time for individual blobs becomes (see Eqs. (29) and (30) of SR03)

$\ell \kappa \rho_{\mathrm{b}} \gtrsim \frac{c}{c_{\mathrm{s}}}$

where $c_{\mathrm{s}}$ is the isothermal sound speed, that is, the optical depth of the blob must be larger than the ratio of light to sound speed. Scaling the variables in the last condition with $\ell=\epsilon^{2} R$, gives,

$1 \lesssim \eta \equiv \epsilon^{2} \kappa R \rho_{\mathrm{b}} \frac{c_{\mathrm{s}}}{c}$,

which defines the function $\eta$. Substituting the density from Eq. (4) in Eq. (10) gives

$1 \lesssim \eta=\frac{\dot{M} \kappa}{4 \pi c \alpha R \epsilon}\left(\frac{c_{\mathrm{s}}}{v_{\mathrm{K}}}\right) \simeq \frac{\dot{M} \kappa}{4 \pi c \alpha R}$,

since $c_{\mathrm{s}} / v_{\mathrm{K}} \simeq \epsilon$. Therefore the condition $\eta \gtrsim 1$ requires

$\dot{M} \gtrsim 4.2 \times 10^{-5} \kappa^{-1}\left(\frac{\alpha}{0.1}\right)\left(\frac{R}{R_{\odot}}\right) M_{\odot} \mathrm{yr}^{-1}$. 
The weak shock temperature $T_{\mathrm{b}}$ will be intermediate between the strong shock temperature

$$
\begin{aligned}
T_{\mathrm{s}} & =\frac{3}{16} \frac{\mu m_{H}}{k} v_{\mathrm{K}}^{2} \simeq 1.4 \times 10^{-9} v_{\mathrm{K}}^{2} \\
& \simeq 2.6 \times 10^{6}\left(\frac{M}{M_{\odot}}\right)\left(\frac{R}{R_{\odot}}\right)^{-1} \mathrm{~K}
\end{aligned}
$$

and the disk temperature, $T_{\mathrm{b}} \simeq 0.05 T_{\mathrm{s}}$, say.

The post-shock temperature of the blobs can be a little higher than $T_{\mathrm{b}}=5 \times 10^{4} \mathrm{~K}$ for YSOs and higher than $T_{\mathrm{b}}=$ $5 \times 10^{6} \mathrm{~K}$ for WD accretors. The cooling time from higher temperatures is shorter. However, what is important for the model is that the shocked blobs don't cool back to the original temperature of $T \sim 10^{4} \mathrm{~K}$ for YSO and $T \sim 10^{6}$ for WD accretors, so that an expansion process of the BL region starts.

For an YSO accretor we take the opacity as given in Eq. (2). Substituting $T_{\mathrm{b}}=5 \times 10^{4} \mathrm{~K}$ gives $\kappa=40\left(\rho / 10^{-7}\right)^{0.88}$. We take the density from Eq. (4) scaled to YSOs, and then substitute the opacity in Eq. (12). The condition for the formation of a strong shock from weakly-shocked small blobs (12) becomes a condition on the accretion rate

$\dot{M}_{\mathrm{b}} \gtrsim 7 \times 10^{-7}\left(\frac{\epsilon}{0.1}\right)^{1.4}\left(\frac{\alpha}{0.1}\right) M_{\odot} \mathrm{yr}^{-1} \quad$ for YSOs.

For WD accretors we take $T_{\mathrm{b}}>5 \times 10^{6}, R=0.01 R_{\odot}$. From Fig. 1 it is clear that the opacity is $\kappa=0.4 \mathrm{~cm}^{2} \mathrm{~g}^{-1}$. The condition of Eq. (11) then reads

$\dot{M}_{\mathrm{b}} \gtrsim 10^{-6}\left(\frac{\alpha}{0.1}\right) M_{\odot} \mathrm{yr}^{-1} \quad$ for $\quad$ WDs,

which is never satisfied in CVs (Warner 1995).

Can a shock be built away from the accreting body, and launch jets there? First from Eqs. (6) and (12) we note that the conditions for accelerating the gas and building a SPLASH are more difficult to meet away from the accreting object. From both equations the accretion rate should increase as $R$. Second, and more important, to build a shock a strong perturbation should occur in the disk. This can be triggered by large gradient in one or more of the physical variables. This is naturally the case in the boundary layer (and perhaps close to the last stable orbit around black holes; this is outside the scope of the present paper). In any case, we cannot rule out the possibility that jets will be launched somewhat away from the boundary layer, up to $\sim 3$ times the boundary layer radius.

\section{Discussion and summary}

In the previous two sections we found the constraints on the mass accretion rate derived using the two conditions for slow radiative cooling derived by SR03. The first condition is that the strongly shocked gas in the BL will cool slowly, such that the thermal pressure will have enough time to accelerate the jet's material. The constraints on the accretion rate, $\dot{M}_{\mathrm{s}}$, are given by Eqs. (7) and (8), for YSO and WD accretors, respectively. The second condition is that weakly shocked blobs in the BL will expand, and disturb the BL in such a way that a strong shock will develop. The constraints on the accretion rate, $\dot{M}_{\mathrm{b}}$, are given by Eqs. (14) and (15), for YSO and WD accretors, respectively. We should stress the following in regard to these constraints. (1) The constraints are accurate to an order of magnitude. This is for several reasons, e.g., the values of $\alpha$ and $\epsilon$ are unknown; the demand on the ratio of the cooling time to acceleration time is given to an order of magnitude; the behavior of the gas, e.g., its opacity and pressure, should be treated more accurately with a full 3D numerical code. (2) The first constraint on $\dot{M}_{\mathrm{s}}$ is generic to the proposed thermallyaccelerated jet model. (3) The second one, on the formation of the disturbances that lead to the formation of strong shocks in the BL, might be less important. This is because other types of disturbances can cause strong shocks to develop in the BL, e.g., MHD instabilities, magnetic eruptions from YSOs, and local thermonuclear events on a WDs.

With these, we note the following. For YSOs (and other main sequence stellar accretors) the two requirements on the two cooling time-scales basically gives the same constraint on the mass accretion rate $\dot{M}$ (YSO) $\gtrsim 10^{-7} M_{\odot} \mathrm{yr}^{-1}$ (Eqs. (7) and (14)). This fits observations, which show jets from YSO accreting at such rates (e.g., Cabrit et al. 1990).

For WDs the two constraints give $\dot{M}_{\mathrm{s}} \gtrsim 10^{-9} M_{\odot} \mathrm{yr}^{-1}$ and $\dot{M}_{\mathrm{b}} \gtrsim 10^{-6} M_{\odot} \mathrm{yr}^{-1}$ (Eqs. (8) and (15)). The second one is very stringent. The highest accretion rates deduced from observations of nova-like systems or dwarf-novae at maximum are two orders of magnitude lower (see e.g., Warner 1995). Taking into account the order of magnitude uncertainty one could maybe get $\dot{M}$ (WD) $\gtrsim 1-3 \times 10^{-7}$. This is compatible with observations of SSXSs and symbiotic systems, and the accretion rate after the outburst of the fast nova V1494 Aql. In this novae Iijima \& Esenoglu (2003) reported the detection of jets. The accretion rate during this stage is estimated to be $\gtrsim 5 \times 10^{-7} M_{\odot} \mathrm{yr}^{-1}$ (Retter, A., private communication 2004). This accretion rate is compatible with our constraint on jets launching. SSXSs are thought to be white dwarfs accreting at rates of $3 \times 10^{-8}-10^{-6} M_{\odot} \mathrm{yr}^{-1}$ from a companion, and sustaining nuclear burning on their surface (e.g., van den Heuvel et al. 1992; Greiner 1996). To maintain a steady nuclear burning the mass accretion rate should be $3 \times 10^{-8}-10^{-6} M_{\odot} \mathrm{yr}^{-1}$, where the upper range is for massive WDs (Nomoto 1982). Fast, $\sim 1000-5000 \mathrm{~km} \mathrm{~s}^{-1}$, collimated outflows have been observed in some SSXSs, RX J0513.9-6951 (Crampton et al. 1996; Southwell et al. 1996), RX J0019.8+2156 (Becker et al. 1998; Quaintrell \& Fender 1998; Tomov et al. 1998), and RX J0925.7-4758 (Motch 1998). These systems teach us that WDs accreting mass at rates much higher than those in cataclysmic variables can blow jets. In RX J0925.7-4758 the high luminosity (Motch 1998) implies an accretion rate of $\sim 10^{-7} M_{\odot} \mathrm{yr}^{-1}$, and the WD radius is $\sim 0.005 R_{\odot}$. With $\epsilon=0.05$ the constraint (Eq. (15)) is $\dot{M}_{\mathrm{b}} \gtrsim 3 \times 10^{-7} M_{\odot} \mathrm{yr}^{-1}$. The accretion rate onto RX J0513.9-6951 is even higher (Southwell et al. 1996). Some symbiotic systems are also known to blow jets (Sokoloski 2004; Brocksopp et al. 2004, and references therein). In these symbiotic systems, WD companions accrete from the wind of red giant branch stars or asymptotic giant branch stars, at relatively high rates. In some of the symbiotic systems which blow jets the WD sustains a quasi-steady nuclear burning, similar to SSXSs; in others, there is no nuclear 
burning (Brocksopp et al. 2004). Still, the mass accretion rate is expected to be high in the later group as well. It is possible that in the later systems the WD are more massive; more massive WD stars require higher mass accretion rates to sustain nuclear burning (Nomoto 1982).

Although no jets have ever been observed in CVs some of them emit winds. P Cygni profiles in resonant UV lines are observed in some very luminous CVs such as the nova-like stars and dwarf novae at outburst maximum. These winds are too cold to be ejected by a thermal mechanisms. They are most probably radiation driven (see e.g., Murray 2002; Proga 2002).

Finally, it is interesting to investigate what kind of condition one obtains when considering ultra-compact objects such as neutron stars and black holes. In such a case it is more convenient to scale Eq. (12) with the Schwarzschild radius $R_{\mathrm{G}}=$ $2 G M / c^{2}$ and the Eddington accretion rate $\dot{M}_{\text {Edd }}=L_{\text {Edd }} / 0.1 c^{2}=$ $2.3 \times 10^{-8} M_{\odot} \mathrm{yr}^{-1}$. One obtains then

$\dot{m} \equiv \frac{\dot{M}}{\dot{M}_{\mathrm{Edd}}} \gtrsim 0.02\left(\frac{\alpha}{0.1}\right)\left(\frac{0.4 \mathrm{~g} \mathrm{~cm}^{-2}}{\kappa}\right) \frac{R}{R_{\mathrm{G}}}$,

which is very close to the value at which low-mass X-ray transients enter (from below) the so called hard/low states associated with the appearance of steady jets (e.g., Fender 2001). Of course there are no boundary layers around accreting black holes so that our model cannot be directly applied to these objects. We will discuss this problem in a future paper.

Acknowledgements. We thank Marek Sikora for his precious remarks on the manuscript. J.P.L. is grateful to Daniel Proga for very useful discussions.

\section{References}

Becker, C. M., Remillard, R. A., Rappaport, S., \& McClintock, J. E. 1998, ApJ, 506, 880

Blandford, R. D., \& Payne, D. G. 1982, MNRAS, 199, 883

Bogovalov, S., \& Tsinganos, K. 2001, MNRAS, 325, 249

Brocksopp, C., Sokoloski, J. L., Kaiser, C., et al. 2004, MNRAS, 347, 430

Cabrit, S., Edwards, S., Strom, S. E., \& Strom, K. M. 1990, ApJ, 354, 687

Crampton, D., Hutchings, J. B., Cowley, A. P., et al. 1996, ApJ, 456, 320

De Villiers, J. P., Hawley, J. F., \& Krolik, J. H. 2004, ApJ, 599, 1238

Fender, R. P. 2001, MNRAS, 322, 31

Ferreira, J. 2002, in Star Formation and the Physics of Young Stars Summer school on Stellar Physics X - EAS Publications Series, vol. 3, ed. J. Bouvier, \& J.-P. Zahn (EDP Sciences)

Ferreira, J., \& Casse, F. 2004, ApJ, 601, L139

Ferreira, J., \& Pelletier, G. 1993, A\&A, 276, 625

Ferreira, J., \& Pelletier, G. 1995, A\&A, 295, 807
Getman, K. V., Feigelson, E. D., Townsley, L., et al. 2002, ApJ, 575, 354

Greiner, J. 1996, Lecture Notes in Physics 472, Supersoft X-Ray Sources (Berlin: Springer)

Heyvaerts, J., \& Norman, C. 1989, ApJ, 347, 1055

Heyvaerts, J., Priest, E. R., \& Bardou, A. 1996, ApJ, 473, 403

Iijima, T., \& Esenoglu, H. H. 2003, A\&A, 404, 997

Königl, A. \& Pudritz, R. E. 2000, in Protostars and Planets IV, ed. V. Mannings, A. P. Boss, \& S. S. Russell (Tucson: Univ. of Arizona Press), 759

Knigge, C., King, A. R., \& Patterson, J. 2000, A\&A, 364, L75

Knigge, C., \& Livio, M. 1998, MNRAS, 297, 1079

Li, L.-X. 2002, ApJ, 564, 108

Li, L.-X., \& Narayan, R. 2004, ApJ, 601, 414

Livio, M. 1999, Phys. Rep., 311, 225

Livio, M. 2000a, in Asymmetrical Planetary Nebulae II: From Origins to Microstructures, ed. J. H. Kastner, N. Soker, \& S. Rappaport (San Francisco: ASP), ASP Conf. Ser., 199, 243

Livio, M. 2000b, in Cosmic Explosions, ed. S. S. Holt, \& W. W. Zhang (American Institute of Physics Conf.), 522, 275

Motch, C. 1998, A\&A, 338, L13

Murray, N. 2002, The Physics of Cataclysmic Variables and Related Objects, ASP Conf. Ser., 261, 308

Nomoto, K. 1982, ApJ, 253, 798

O’Brien, T. J., \& Cohen, J. G. 1998, ApJ, 498, L59

Proga, D. 2002, Mass Outflow in Active Galactic Nuclei: New Perspectives, ASP Conf. Ser., 255, 309

Quaintrell, H., \& Fender, R. P. 1998, A\&A, 335, L17

Pringle, J. E. 1981, ARA\&A, 19, 137

Pringle, J. E., \& Savonije, G. J. 1979, MNRAS, 187, 777

Rogers, F. J., \& Iglesias, C. A. 1992, ApJS, 79, 507

Seaton, M. J., Yan, Y., Mihalas, D., \& Pradhan, A. K. 1994, MNRAS, 266, 805 (http://vizier.u-strasbg.fr/cgi-bin/OP/S92)

Shahbaz, T., Livio, M., Southwell, K. A., \& Charles, P. A. 1997, ApJ, 484, L59

Shu, F. H., Lizano, S., Ruden, S., \& Najita, J. 1988, ApJ, 328, L19

Shu, F. H., Najita, J. R., Shang, H., \& Li, Z.-Y. 2000, in Protostars and Planets IV, ed. V. Mannings, A. P. Boss, \& S. S. Russell (Tucson: Univ. of Arizona Press), 789

Shu, F. H., Ruden, S. P., Lada, C. J., \& Lizano, S. 1991, ApJ, 370, L31

Soker, N., \& Regev, O. 2003, A\&A, 406, 603 (SR03)

Sokoloski, J. L. 2004, Journal of the American Association of Variable Star Observers (AAVSO) [arXiv: astro-ph/0403004], in press

Southwell, K. A., Livio, M., Charles, P. A., O’Donoghue, D., \& Sutherland, W. J. 1996, ApJ, 470, 1065

Tomov, T., Munari, U., Kolev, D., Tomasella, L., \& Rejkuba, M. 1998, A\&A, 333, L67

Torbett, M. V. 1984, ApJ, 278, 318

Torbett, M. V., \& Gilden, D. L. 1992, A\&A, 256, 686 (TG)

van den Heuvel, E. P. J., Bhattacharya, D., Nomoto, K., \& Rappaport, S. A. 1992, A\&A, 262, 97

Warner, B. 1995, Cambridge Astrophysics Series (Cambridge, New York: Cambridge University Press) 